\title{
BMJ Open Sex differences in macronutrient intake and adherence to dietary recommendations: findings from the UK Biobank
}

\author{
Elizabeth Bennett, ${ }^{1,2}$ Sanne A E Peters, ${ }^{1}$ Mark Woodward ${ }^{1,3,4}$
}

To cite: Bennett E, Peters SAE, Woodward M. Sex differences in macronutrient intake and adherence to dietary recommendations: findings from the UK Biobank. BMJ Open 2018:8:e020017. doi:10.1136/ bmjopen-2017-020017

- Prepublication history and additional material for this paper are available online. To view these files, please visit the journal online (http://dx.doi. org/10.1136/bmjopen-2017020017).

Received 8 0ctober 2017 Revised 14 February 2018 Accepted 23 February 2018

\section{Check for updates}

${ }^{1}$ The George Institute for Global Health, University of Oxford, Oxford, UK

${ }^{2}$ School of Medicine, Faculty of Medicine, Nursing and Health Sciences, Flinders University, Adelaide, South Australia, Australia

${ }^{3}$ The George Institute for Global Health, University of New South Wales, Sydney, New South

Wales, Australia

${ }^{4}$ Department of Epidemiology, John Hopkins University, Baltimore, Maryland, USA

Correspondence to Dr Sanne A E Peters; sanne.peters@georgeinstitute. ox.ac.uk

\section{ABSTRACT}

Objectives To characterise sex differences in macronutrient intakes and adherence to dietary recommendations in the UK Biobank population. Design Cross-sectional population-based study. Setting UK Biobank Resource.

Participants 210106 (52.5\% women) individuals with data on dietary behaviour.

Main outcome measures Women-to-men mean differences in nutrient intake in grams and as a percentage of energy and women-to-men ORs in non-adherence, adjusting for age, socioeconomic status and ethnicity. Results There were sex differences in energy intake and distribution. Men had greater intakes of energy and were less likely to have energy intakes above the estimated average requirement compared with women. Small, but significant, sex differences were found in the intakes of all macronutrients. For all macronutrients, men had greater absolute intakes while women had greater intakes as a percentage of energy. Women were more likely to have intakes that exceeded recommendations for total fat, saturated fat and total sugar. Men were less likely to achieve the minimum recommended intakes for protein, polyunsaturated fat and total carbohydrate. Over $95 \%$ of men and women were non-adherent to fibre recommendations. Sex differences in dietary intakes were moderated by age and to some extent by socioeconomic status.

Conclusions There are significant sex differences in adherence to dietary recommendations, particularly for sugar. However, given the increased focus on food groups and dietary patterns for nutritional policy, these differences alone may not be sufficient for policy and health promotion. Future studies that are able to explore the sex differences in intakes of different food groups that are risk factors for diet-related diseases are warranted to improve the current understanding of the differential impact of diet on health in women and men.

\section{INTRODUCTION}

Poor quality diet is a leading cause of morbidity and mortality worldwide and the leading risk factor for non-communicable diseases (NCDs). ${ }^{1-3}$ Worldwide, the burden of NCD is expected to further increase with

\section{Strengths and limitations of this study}

- The availability of 24 hours recall data on dietary behaviour from over 200000 individuals permits a comprehensive evaluation of sex differences in dietary behaviours.

- Self-reported dietary data can be subject to recall bias, social desirability bias and under-reporting, which may be sex differential.

- Given that over $90 \%$ of the participants in the UK Biobank are Caucasian, the present analyses cannot be generalised to other ethnic groups.

- The cross-sectional nature of our analyses precluded the examination of associations between dietary behaviours and health outcomes.

population ageing and increasing rates of obesity, together with other diet-related risk factors. Even modest dietary changes are associated with meaningful reductions in cardiovascular disease morbidity and mortality, type 2 diabetes, specific cancer sites and their major risk factors, including hypercholesterolaemia, hypertension and obesity. ${ }^{4-9}$

In the UK and elsewhere, adults continue to consume too much saturated fat, sugar, red and processed meat and sugar-sweetened beverages, while intakes of fruit, vegetables, oily fish and fibre are insufficient. ${ }^{10}$ Recent analyses indicate that there is little or no evidence of change in the problematic nutrition patterns in UK adults, suggesting that current strategies to improve diet are insufficient at a population level. ${ }^{10-12}$

It is widely recognised that sex differences in dietary intakes and dietary behaviour exist; however these are not well characterised. ${ }^{13-18}$ Previous analyses of dietary intakes in the UK have relied on estimates from the National Diet and Nutrition Survey; a small study limited by well-documented under-reporting and estimates from expenditure intakes, which may not accurately reflect actual consumption. ${ }^{1019}$ 
These national estimates did not examine sex differences as a primary outcome nor their variation across age and sociodemographic subpopulations.

Optimising dietary habits to improve population health requires systematically identified and evaluated data. ${ }^{20}$ Thus, a better understanding of dietary patterns of nutrient consumption by sex is crucial to establish priorities for dietary guidelines and to inform, design and implement strategies for reducing diet-related disease. Identification of sex disparities in dietary intakes and adherence to dietary guidelines can help to subsequently facilitate improvement in population nutrition strategies.

In this study, we therefore aimed to characterise sex differences in macronutrient intakes and adherence to dietary recommendations in the UK Biobank, which includes the largest dietary survey in the UK to date. ${ }^{21}$

\section{METHODS}

\section{Study population}

Cross-sectional data were used from the UK Biobank, a large-scale, prospective cohort study among 502712 men and women aged 40-69 at baseline. ${ }^{21}$ Between 2006 and 2010, participants attended 1 of the 22 centres across the UK for detailed baseline assessment that involved collection of questionnaire data, physical measurements, and biological samples. All participants provided electronic informed consent.

\section{Dietary data collection}

Information about dietary behaviour was collected using 24-hour dietary recall questionnaires. ${ }^{22}$

The questionnaires contained questions on the intake of over 200 food and drink items, grouped into broad categories, over the last 24 hours. Where the foods did not match the items listed exactly, participants were encouraged to try and choose a food or a combination of foods that most closely resembles what they had and to not duplicate food items. Participants were asked whether what they ate and drank yesterday was typical, and if not, the reason; and whether they routinely followed a special diet, and if so, what kind of diet. So that the replies could be coded automatically to provide estimated daily nutrient intake, open-ended questions were avoided, although some free text boxes were available for use when the options listed did not cover a particular food item. The email invitations were issued on specific days of the week to capture variations in intake between week days and weekend days. For the first and second round of email invitations, participants were allowed 3 days to complete the questionnaire, after which time the link had expired; this was extended to 14 days for the third and fourth round of email invitations. These questionnaires were first introduced as part of the assessment visit towards the end of the recruitment phase,and were also completed remotely via the internet for those participants who have provided UK Biobank with email addresses.
Table 1 Recommended dietary intake of energy and macronutrients for adults in the UK

\section{Recommended daily intake}

\begin{tabular}{ll}
\hline Energy & \\
\multicolumn{1}{|l}{ Men } & $<10460 \mathrm{~kJ}$ \\
\hline Women & $<8363 \mathrm{~kJ}$ \\
\hline Fat & \\
\hline Total fat & $<35 \% \mathrm{El}$ \\
Saturated fat & $<11 \% \mathrm{El}$ \\
Polyunsaturated fat & $6 \%-11 \% \mathrm{El}$ \\
Carbohydrates & \\
Carbohydrate & $>50 \% \mathrm{El}$ \\
Total sugars & \\
$\quad$ Men & $<120 \mathrm{~g}$ \\
$\quad$ Women & $<90 \mathrm{~g}$ \\
Fibre & $\geq 30 \mathrm{~g}$ \\
\hline Protein & $0.75 \mathrm{~g}$ per kg body weight \\
\hline
\end{tabular}

El, energy intake.

\section{Nutritional data}

The nutrient intakes for each participant were calculated using the UK food composition database. ${ }^{23}$ Each food and beverage listed in the questionnaire was assigned a portion size based on the unit listed in the questionnaire. ${ }^{24}$ The percentage of energy intake of macronutrients was determined by, first, multiplying the consumption by the metabolisable energy conversion factors and, second, dividing the resulting kilojoule contribution by the total energy intake.

\section{Adherence to dietary guidelines}

Adherence to dietary guidelines was assessed by comparing the intakes of macronutrients to recommendations from the UK government guidelines (table 1). ${ }^{1725}{ }^{26}$ Energy intakes more than 4 SD from the mean were considered implausible, thus excluding 547 women and 413 men. ${ }^{27} 28$

\section{Statistical methods}

Baseline characteristics are presented as means (SD) for continuous variables and as percentages for categorical variables. General linear models were used to obtain the women-to-men differences and 95\% CIs in mean energy and macronutrient intake. Logistic regression analyses were used to compute the women-to-men ORs for adherence to recommended dietary intakes. All analyses were adjusted for age, ethnicity, and socioeconomic status (SES), measured using the Townsend deprivation index, a measure of material deprivation within a population. Five SES groups were defined using the quintiles of the Townsend deprivation index in England from the 2001 Census. $^{29}$ Subgroup analyses were performed by age group, SES and body mass index (BMI). To assess the impact of inherent differences between women and men in body weight on energy and macronutrient intake, sensitivity analyses, overall and by age group and SES were 
Table 2 Baseline characteristics of study participants

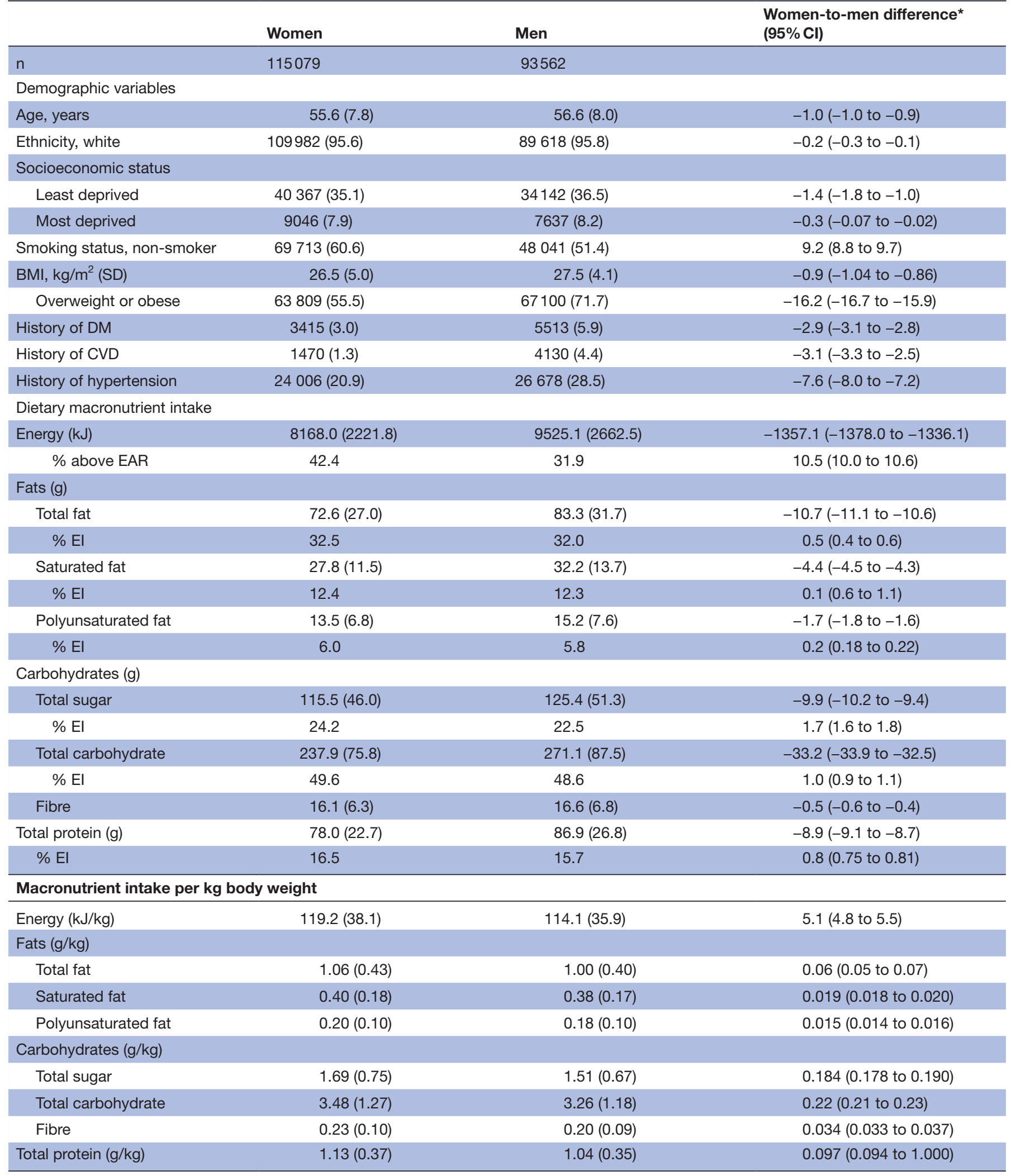

Continuous variables are expressed as means and standard deviation while categorical variables are expressed as a $\mathrm{n}$ and percentages.

*Sex difference calculated as Women-men, adjusted for age, socioeconomic status and ethnicity.

BMI, body mass index; CVD, cardiovascular disease; DM, diabetes mellitus; EAR, estimated average requirement; El, energy intake. 

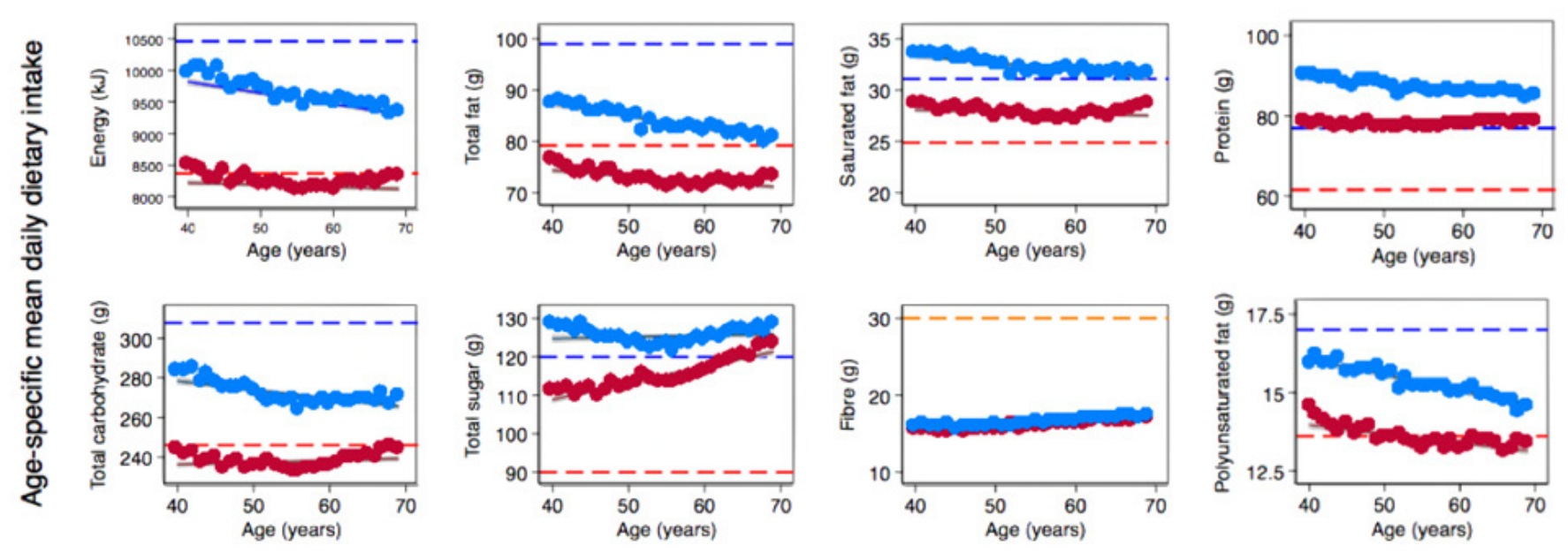

Figure 1 Energy $(\mathrm{kJ})$ and macronutrient $(\mathrm{g})$ intakes per year of age by sex. The points represent the age-specific mean daily dietary intake (blue for men, red for women) at different ages. The dashed lines represent the recommended daily intake (blue for men, red for women, orange for men and women). The solid lines represent the linear model relating dietary intake to age (blue for men, red for women).

conducted in which the absolute intake of each nutrient was divided by the participant's body weight. All analyses were conducted using Stata V.14.0.

\section{RESULTS}

Of the 210106 participants with 24 hours dietary recall data, the mean age at recruitment was 56 years and $55 \%$ was women. On average, men were more likely to have ever smoked, to be overweight or obese, and to have a history of diabetes, cardiovascular disease, and hypertension (table 2).

\section{Energy and macronutrient intakes}

Men had a significantly higher energy and macronutrient intake than women, with a mean difference in total energy intake of $1358 \mathrm{~kJ}$ per day. In contrast, when standardised by body weight, women's energy consumption was higher than in men. Similarly, $42 \%$ of women consumed more energy than recommended compared with $32 \%$ of men.

Intake of all macronutrients as a percentage of energy intake were greater for women than for men, with the largest difference being for total sugar intake $(22.5 \%$ of total energy intake in men compared with $24.2 \%$ in women). Women-to-men differences in energy and macronutrient intake decreased with age (figure 1 and online supplementary eTable 1 ). Total energy intake was $1555 \mathrm{~kJ}$ greater in men than women in participants aged 40-44 years compared with $1157 \mathrm{~kJ}$ in participants aged 64-69 years old. The differences in total fat, saturated fat and polyunsaturated intakes decreased by $3.8 \mathrm{~g}$, $1.6 \mathrm{~g}$ and $0.5 \mathrm{~g}$, respectively, between the youngest and oldest participants ( $p$ value for interaction between sex and age $<0.01$ for all macronutrients). The differences in carbohydrate, sugar, fibre and protein intakes decreased

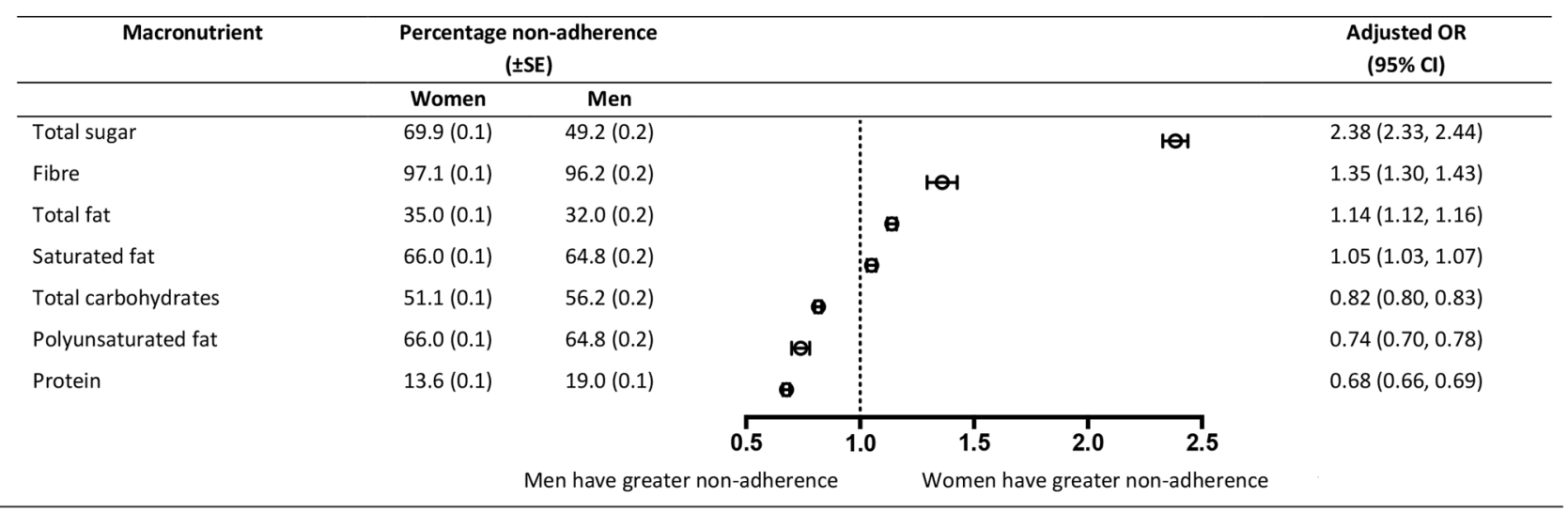

OR: odds ratio; SE: standard error; $95 \% \mathrm{Cl}$ : $95 \%$ confidence interval

Figure 2 Adjusted ORs (women vs men) for non-adherence to macronutrient intake recommendations. Analyses are adjusted for age, socioeconomic status and ethnicity. Points represent ORs and horizontal lines indicate the corresponding $95 \%$ Cls. 

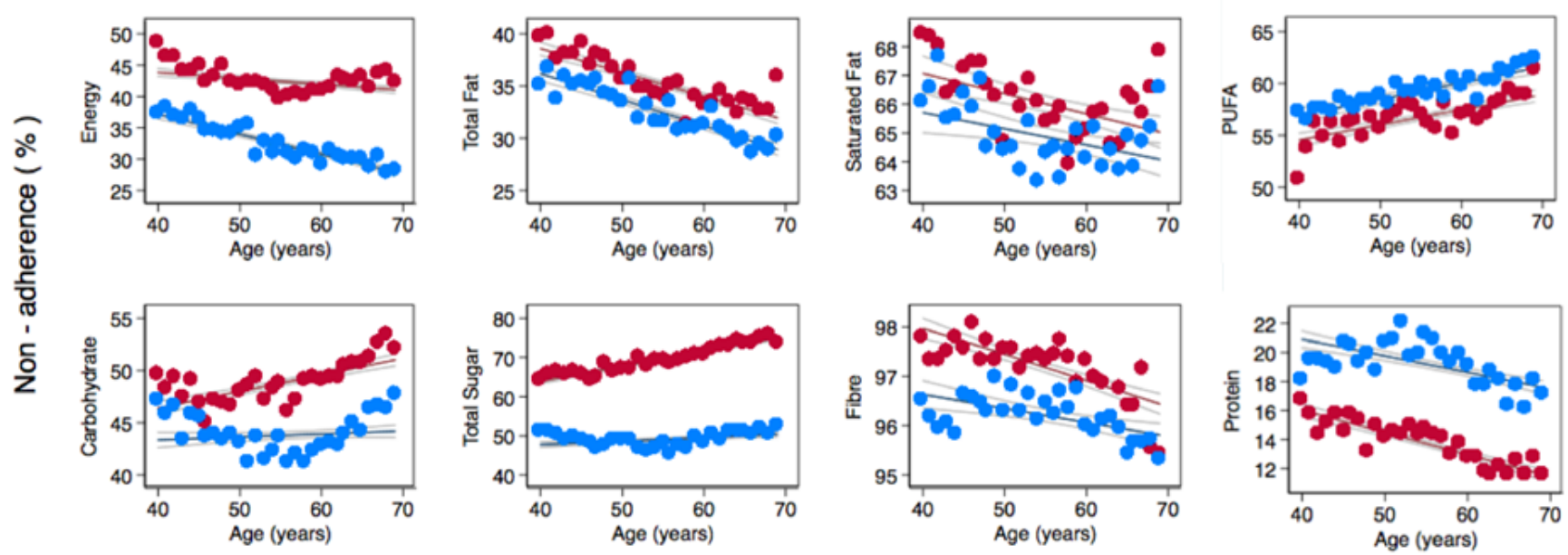

Figure 3 Non-adherence to dietary recommendations per year of age by sex. The points represent the age-specific nonadherence (blue for men, red for women) at different ages. The solid lines represent the linear model relating non-adherence to age (blue for men, red for women).

by $15.1 \mathrm{~g}, 11 \mathrm{~g}, 0.3 \mathrm{~g}$ and $4.1 \mathrm{~g}$, respectively, between the youngest and oldest participants ( $p$ values for interaction $<0.01)$. Sex differences in dietary intakes did not differ materially by SES, with the exceptions of sugar and protein intake (see online supplementary eTable 2 ). The women-to-men difference in sugar intake was $-8.5 \mathrm{~g}$ in the least deprived group and $-13.3 \mathrm{~g}$ in the most deprived group. The sex difference in protein intake was $-8.2 \mathrm{~g}$ in the least deprived group and $-10.4 \mathrm{~g}$ in the most deprived group. Sex differences in energy and macronutrient intake were smaller among obese individuals, compared with those with a healthy BMI (see online supplementary eTable 3). The sex difference in total fat and sugar intake, respectively, were $-11.5 \mathrm{~g}$ and $-13.2 \mathrm{~g}$ among those with a healthy BMI compared with $-9.6 \mathrm{~g}$ and $-5.9 \mathrm{~g}$ among those with obesity.

\section{Adherence to dietary guidelines}

Non-adherence to macronutrient intake recommendations was high in both men and women for most macronutrients. More than half of all participants did not adhere to recommendations for the intake of total sugar, fibre, saturated fat, carbohydrate and polyunsaturated fat (figure 2). Women were significantly more likely than men to exceed recommended intakes of total sugar (OR (95\% CI): 2.38 (2.33 to 2.44)), total fat (1.35 (1.30 to $1.43)$ ) and saturated fat (1.05 (1.03 to 1.07)). Men were more likely than women to have intakes of polyunsaturated fat $(0.74(0.70 ; 0.78))$, carbohydrates $(0.82,(0.80$ to $0.83))$ and protein $(0.68(0.66$ to 0.69$))$, that were under the recommended amounts. Women were significantly more likely than men to have fibre intake below the recommended amount (1.35 (1.30 to 1.43)), although more than $96 \%$ of men failed to eat a recommended amount of fibre.

Non-adherence to dietary guidelines varied by age in both men and women, with evidence that the magnitude of the women-to-men difference in non-adherence increased with age for total carbohydrate and total sugar (figure 3 and online supplementary eTables 4 and 5). For fibre, the sex difference in non-adherence was lower among older than among younger participants. The sex difference in non-adherence varied by SES for all macronutrients, except polyunsaturated fat and total carbohydrate (see online supplementary eTables 6 and 7). Sex differences in non-adherence to dietary guidelines varied across BMI categories for all macronutrients, except for fats (see online supplementary eTables 8 and 9). When standardised for body weight, sex differences in intake of energy, total carbohydrate and total sugar tended to increase with age and SES (see online supplementary eTables 10 and 11).

\section{DISCUSSION}

This large study of over 200000 men and women from the UK Biobank showed that there are some notable sex differences in macronutrient intakes and adherence to dietary recommendations. While adherence to recommended dietary guidelines was suboptimal in both sexes, women were significantly more likely than men to exceed recommended intakes of total sugar, total fat and saturated fat, whereas men were more likely to have intakes under the recommended amounts of polyunsaturated fat, carbohydrate and protein. Sex differences in energy and macronutrient intakes varied by age and SES, suggesting the need for tailored interventions to optimise dietary behaviour in men and women across the life course.

The sex differences in energy intake reported here are consistent with the well-established sex differences in energy intake due to differences in physiological composition. ${ }^{26}$ The National Diet and Nutrition Survey (NDNS), an annual nationally representative survey of 
500 adults, found that men have greater absolute intakes of all macronutrients, as was found in this study. The most recent NDNS data found that men consume a greater percentage of total energy from sugar than women, while women consume more fat, saturated fat, carbohydrate, and protein as percentage of total energy than men. Apart from sugar intakes, these findings are consistent with the results of this study where women had greater intakes of all macronutrients. Several analyses of NDNS data have found significant under-reporting, with a higher rate of under-reporting of energy intake in women than men. ${ }^{19}{ }^{30}$ Hence, our observation that more women than men exceeded their estimated average energy requirement may be an underestimate of the true sex difference in excess energy intake.

Overall, women were more likely to exceed macronutrient recommendations that were maximum amounts (ie, total fat, saturated fat, and sugar) while men were less likely to achieve macronutrient recommendations that were minimum amounts (ie, carbohydrate, protein, and polyunsaturated fat). This is contrary to the general assumption that women would be more likely to adhere to dietary recommendations and have a higher quality diet. ${ }^{31-33}$ Most notably, over 20\% more women than men exceeded the recommended daily intake of total sugar. There are currently no studies in the UK that examine adherence to dietary guidelines, so it is difficult to place these results in context of the current literature. However, these findings suggest different areas of focus may be useful in targeting adherence to dietary guidelines in men and women, particularly for sugar. Several studies have shown that women have a higher intake of sugar compared with men. ${ }^{34-36}$ A potential explanation for this difference may be that women in the UK consume more fruit than men. ${ }^{10}$ Fruit is a source of natural sugar and therefore would result in increased total sugar without increasing free or added sugar intake. However, there is also evidence that women consume more foods high in added sugars than men, such as cookies, chocolate, and ice cream. ${ }^{37-39}$ A recent UK governmental report found there is robust evidence that adherence to sugar recommendations would result in substantial cost and health benefits. ${ }^{40}$ Therefore, future studies should examine sex differences in dietary sources of sugar to identify key foods for policy targeting.

Although this research provides valuable insights into sex differences in dietary behaviour in the UK, there is a move in nutritional research and policy towards focusing on the food groups and diet patterns, as opposed to individual macronutrients. ${ }^{41}$ Sex differences in food groups, dietary patterns, and overall dietary quality were not examined in this study, as this information was not available at the time of analysis. Furthermore, this research does not consider micronutrients, within-individual correlations of different diet components, or possible synergistic or antagonistic effects of nutrients when consumed together. Future studies should investigate these components as this information may provide a more detailed and holistic analysis of sex differences in diet and would be in line with an increased focus on dietary patterns in nutritional interventions. ${ }^{41}$ In particular, further exploration of the dietary sources (ie, fruit and vegetables or processed foods) of sugar will be vital in understanding the association between sex differences in sugar intake.

In conclusion, adherence to UK dietary recommendations for macronutrient intake is suboptimal, particularly for fibre, and varies considerably by sex, particularly for sugar. Given the increased focus on food groups and dietary patterns for nutritional policy, these differences alone may not be suitable for policy and health promotion. Future studies that are able to explore the sex differences in intakes of different food groups that are risk factors for NCDs are warranted to improve the current understanding of the differential impact of diet on health in women and men.

Acknowledgements This research has been conducted using the UK Biobank Resource (application 2495).

Contributors EB performed statistical analyses and wrote the first draft of the article. MW and SAEP conceived the research and produced the final version of the article.

Funding SAEP is supported by a UK Medical Research Council Skills Development Fellowship (MR/P014550/1). MW is supported by an Australian National Health and Medical Council Principal Research Fellowship.

Competing interests None declared.

Patient consent Obtained.

Ethics approval UK Biobank has obtained Research Tissue Bank approval from its governing research ethics committee, as recommended by the National Research Ethics Service. No separate ethics approval was required. Permission to use the UK Biobank Resource was approved by the Access Sub-Committee of the UK Biobank Board.

Provenance and peer review Not commissioned; externally peer reviewed. Data sharing statement The UK Biobank holds the data used in this article.

Open Access This is an Open Access article distributed in accordance with the Creative Commons Attribution Non Commercial (CC BY-NC 4.0) license, which permits others to distribute, remix, adapt, build upon this work non-commercially, and license their derivative works on different terms, provided the original work is properly cited and the use is non-commercial. See: http://creativecommons.org/ licenses/by-nc/4.0/

(C) Article author(s) (or their employer(s) unless otherwise stated in the text of the article) 2018. All rights reserved. No commercial use is permitted unless otherwise expressly granted.

\section{REFERENCES}

1. Murray CJ, Atkinson C, Bhalla K, et al. The state of US health, 1990-2010: burden of diseases, injuries, and risk factors. JAMA 2013;310:591-608.

2. Forouzanfar MH, Alexander L, Anderson HR, et al. Global, regional, and national comparative risk assessment of 79 behavioural, environmental and occupational, and metabolic risks or clusters of risks in 188 countries, 1990-2013: a systematic analysis for the Global Burden of Disease Study 2013. Lancet 2015;386:2287-323.

3. Lim SS, Vos T, Flaxman AD, et al. A comparative risk assessment of burden of disease and injury attributable to 67 risk factors and risk factor clusters in 21 regions, 1990-2010: a systematic analysis for the Global Burden of Disease Study 2010. Lancet 2012;380:2224-60.

4. Mozaffarian D, Hao T, Rimm EB, et al. Changes in diet and lifestyle and long-term weight gain in women and men. $N$ Engl J Med 2011;364:2392-404.

5. Mozaffarian D, Wilson PW, Kannel WB. Beyond established and novel risk factors: lifestyle risk factors for cardiovascular disease. Circulation 2008;117:3031-8. 
6. Danaei G, Ding EL, Mozaffarian D, et al. The preventable causes of death in the United States: comparative risk assessment of dietary, lifestyle, and metabolic risk factors. PLoS Med 2009;6:e1000058.

7. Mozaffarian D, Appel LJ, Van Horn L. Components of a cardioprotective diet: new insights. Circulation 2011;123:2870-91.

8. Mozaffarian D, Capewell S. United Nations' dietary policies to prevent cardiovascular disease. BMJ 2011;343:d5747.

9. Lloyd-Jones DM, Hong Y, Labarthe D, et al. Defining and setting national goals for cardiovascular health promotion and disease reduction: the American Heart Association's strategic impact goal through 2020 and beyond. Circulation 2010;121:586-613.

10. Public Health England. National Diet and Nutrition Survey: results from years 5-6 combined of the rolling programme for 2012 and 2013 to 2013 and 2014). London, UK: Public Health England, 2016.

11. Department for Environment Food and Rural Affairs. Family food 2014. London, UK: Department for Environment, Food and Rural Affairs, 2015

12. Jebb SA, Aveyard PN, Hawkes C. The evolution of policy and actions to tackle obesity in England. Obes Rev 2013;14(Suppl 2):42-59.

13. Imamura F, Micha R, Khatibzadeh S, et al. Dietary quality among men and women in 187 countries in 1990 and 2010: a systematic assessment. Lancet Glob Health 2015;3:e132-e142.

14. Institute of Medicine. Women's health. Report of the public health service task force on women's health issues. Public Health Rep 1985; 100:73-106.

15. Legato M, Bilezikian JP. Prinicples of gender-specific medicine. Amsterdam, Boston: Elsevier Academic Press, 2009.

16. Schwab K, Zahidi S, Papoutsakis D, et al. The global gender gap report. Geneva: World Economic Forum, 2009.

17. Scientific Advisory Committee on Nutrition. Carbohydrates and health. London: TSO, 2015.

18. Wizemann T, Pardue ML. Exploring the biological contributions to human health: does sex matter? Washington DC: Institute of MEdicine 2001.

19. Rennie KL, Coward A, Jebb SA. Estimating under-reporting of energy intake in dietary surveys using an individualised method. Br J Nutr 2007:97:1169-76.

20. Micha R, Khatibzadeh S, Shi P, et al. Global, regional and national consumption of major food groups in 1990 and 2010: a systematic analysis including 266 country-specific nutrition surveys worldwide. BMJ Open 2015;5:e008705

21. UK Biobank. Protocol for a large-scale prospective epidemiological resource. $2007 \mathrm{http} / / \mathrm{www}$ ukbiobank ac uk/wp-content/ uploads/2011/11/UK-Biobank-Protocol pdf

22. Liu B, Young H, Crowe FL, et al. Development and evaluation of the Oxford WebQ, a low-cost, web-based method for assessment of previous $24 \mathrm{~h}$ dietary intakes in large-scale prospective studies. Public Health Nutr 2011;14:1998-2005.

23. Food Standards Agency. McCane and Widdowson's the composition of foods. 6 edn. Cambridge: Royal Society of Chemistry, 2002.
24. Ministy of Agriculture, Fisheries, and Food. Food portion sizes. 2 edn London: HMSO, 1993.

25. Department of Health. Dietary reference for food energy and nutrients for the United Kingdom. London: HMSO, 1991.

26. Scientific Advisory Committee on Nutrition. Dietary reference values for energy. London: TSO, 2011.

27. Mendez MA, Popkin BM, Buckland G, et al. Alternative methods of accounting for underreporting and overreporting when measuring dietary intake-obesity relations. Am J Epidemiol 2011;173:448-58.

28. Williett WC. Implications of total energy intake for epidemiologic analyses. Nutritional epidemiology. New York: Oxford University Press, 2012:260-86.

29. Townsend material deprivation score for output areas in England based on Census 2001. 2004 http://www.apho.org.uk/resource/item. aspx?RID=47506.

30. Public Health England. National Diet and Nutrition Survey: results from years 1-4 combined of the rolling programme (2008 and 2009 to 2011 and 2012. London, UK: Public Health England, 2014.

31. Arganini C, Saba A, Comitato R, et al. Public health-social and behavioural health-gender differences in food choice and dietary intake in modern Western societies. Shanghai, China: InTech, 2012.

32. Bates CJ, Prentice A, Finch S. Gender differences in food and nutrient intakes and status indices from the National Diet and Nutrition Survey of people aged 65 years and over. Eur J Clin Nutr 1999;53:694-9.

33. Marino M, Masella R, Bulzomi P, et al. Nutrition and human health from a sex-gender perspective. Mol Aspects Med 2011;32:1-70.

34. Millen BE, Quatromoni PA, Franz MM, et al. Population nutrient intake approaches dietary recommendations: 1991 to 1995 Framingham Nutrition Studies. J Am Diet Assoc 1997;97:742-9.

35. Vadiveloo M, Scott M, Quatromoni P, et al. Trends in dietary fat and high-fat food intakes from 1991 to 2008 in the Framingham Heart Study participants. Br J Nutr 2014;111:724-34.

36. Vitale M, Masulli M, Cocozza S, et al. Sex differences in food choices, adherence to dietary recommendations and plasma lipid profile in type 2 diabetes - the TOSCA.IT study. Nutr Metab Cardiovasc Dis 2016;26:879-85.

37. Kanter R, Caballero B. Global gender disparities in obesity: a review. Adv Nutr 2012;3:491-8.

38. Wansink B, Cheney MM, Chan N. Exploring comfort food preferences across age and gender. Physiol Behav 2003;79:739-47.

39. Wardle J, Haase AM, Steptoe A, et al. Gender differences in food choice: the contribution of health beliefs and dieting. Ann Behav Med 2004;27:107-16.

40. Public Health England. Sugar reduction: the evidence for action. London, UK: Public Health England, 2015.

41. Dietary Guidelines Advisory Committee. Scientific Report of the 2015 Dietary Guidelines Advisory Committee: food and nutrient intakes, and health-current status and trends: Dietary Guidelines Advisory Committee, 2015. 